\title{
Linkage and potential association of obesity-related phenotypes with two genes on chromosome 12q24 in a female dizygous twin cohort
}

Scott G Wilson ${ }^{1}$, Gail Adam ${ }^{2,8}$, Maria Langdown ${ }^{2}$, Rikard Reneland ${ }^{2}$, Andreas Braun ${ }^{2}$, Toby Andrew ${ }^{3}$, Gabriela L Surdulescu ${ }^{3}$, Maria Norberg ${ }^{2,8}$, Frank Dudbridge ${ }^{4}$, Peter W Reed ${ }^{5}$, Philip N Sambrook ${ }^{6}$, Patrick W Kleyn ${ }^{7}$ and Tim D Spector ${ }^{* 3}$

${ }^{1}$ Department of Endocrinology \& Diabetes, Sir Charles Gairdner Hospital, Nedlands, Australia; ${ }^{2}$ Sequenom Inc., San Diego, CA, USA; ${ }^{3}$ Twin \& Genetic Epidemiology Research Unit, St Thomas' Hospital, London, UK; ${ }^{4}$ MRC Biostatistics Unit, Cambridge, UK; ${ }^{5}$ SignaGen, Rotorua, New Zealand; ${ }^{6}$ Institute of Bone and Joint Research, University of Sydney, Sydney, Australia; ${ }^{7}$ Broad Institute of MIT and Harvard, Cambridge, MA, USA

Obesity is a multifactorial disorder with a complex phenotype. It is a significant risk factor for diabetes and hypertension. We assessed obesity-related traits in a large cohort of twins and performed a genome-wide linkage scan and positional candidate analysis to identify genes that play a role in regulating fat mass and distribution in women. Dizygous female twin pairs from 1094 pedigrees were studied (mean age $47.0 \pm 11.5$ years (range 18-79 years)). Nonparametric multipoint linkage analyses showed linkage for central fat mass to $12 \mathrm{q} 24(141 \mathrm{cM})$ with LOD 2.2 and body mass index to $8 \mathrm{q} 11(67 \mathrm{cM})$ with LOD 1.3, supporting previously established linkage data. Novel areas of suggestive linkage were for total fat percentage at 6q12 (LOD 2.4) and for total lean mass at 2q37 (LOD 2.4). Data from follow-up fine mapping in an expanded cohort of 1243 twin pairs reinforced the linkage for central fat mass to $12 q 24$ (LOD 2.6; $143 \mathrm{cM}$ ) and narrowed the -1 LOD support interval to $22 \mathrm{cM}$. In all, 45 single-nucleotide polymorphisms (SNPs) from 26 positional candidate genes within the 12q24 interval were then tested for association in a cohort of 1102 twins. Single-point Monks-Kaplan analysis provided evidence of association between central fat mass and SNPs in two genes - PLA2G1B $(P=0.0067)$ and $P 2 R X 4(P=0.017)$. These data provide replication and refinement of the 12q24 obesity locus and suggest that genes involved in phospholipase and purinoreceptor pathways may regulate fat accumulation and distribution.

European Journal of Human Genetics (2006) 14, 340-348. doi:10.1038/sj.ejhg.5201551; published online 4 January 2006

Keywords: twins; linkage; association; positional candidate; central obesity; polymorphism; SNP

\footnotetext{
*Correspondence: Professor TD Spector, Twin and Genetic Epidemiology Research Unit, St Thomas' Hospital, Lambeth Palace Road, London SE1 7EH, UK. Tel: + 44207960 5557; Fax + 44207922 8234;

E-mail: tim.spector@kcl.ac.uk

${ }^{8}$ Current address: ARA Life Science, Technosite, Smedsgränd 3, 753 20, Uppsala, Sweden

Received 12 May 2005; revised 25 October 2005; accepted 27 October 2005; published online 4 January 2006
}

Introduction

Obesity is a major public health problem. Estimates suggest that 315 million people are obese ${ }^{1}$ and the number is increasing. At the basic level, obesity is always due to greater energy intake than is expended, but the interaction of genetic and environmental factors that regulate both accumulation and distribution of body fat is complex. Recent studies of twins from our cohort, ${ }^{2}$ and others, ${ }^{3,4}$ have shown that total and central fat mass are responsible for most of the additional circulating inflammatory 
markers and cytokines that lead to increased heart disease. Determinants of fat accumulation include basal and sleeping metabolic rate, thermogenesis and the thermic effect of food, physical activity, efficiency of nutrient absorption by the gut and hormonal status, ${ }^{5}$ which are all regulated differently depending on genetic make-up. Studies of twins have examined obesity-related trait heritability in both sexes, and values generally range from 0.47 to $0.86,{ }^{6-9}$ with slightly lower estimates yielded by family studies. ${ }^{10-12}$ A number of genome-wide linkage studies have been performed for obesity-related traits to define the location of quantitative trait loci (QTLs) that regulate obesity phenotypes. ${ }^{13}$ Among these, a role for the SLC6A14 gene in appetite control and obesity represents an important demonstration of the utility of this approach. ${ }^{14-16}$ Replication of other established linkage data remains a priority. In addition, while obesity-related phenotypes such as body mass index (BMI) have been considered previously, central fat mass is a core component of the metabolic syndrome and a stronger predictor of insulin resistance than trunk, limb or total fat. ${ }^{17,18}$ Cardiovascular disease in women is also known to be strongly associated with central obesity, $8,17,19,20$ and therefore identification of genes that regulate central fat mass is particularly important.

This report describes genome-wide linkage scan data from a large cohort of female dizygous twins, and the use of a positional candidate approach to find genes influencing central obesity.

\section{Materials and methods}

\section{Subjects and clinical assessment}

Initial twin cohort The St Thomas' UK twin registry (TwinsUK) was used to identify Caucasian dizygous twin sister pairs between 18 and 80 years of age, unselected for any trait or disease. This was a volunteer sample and none of the participants was aware of the specific hypothesis to be studied. Subjects were assessed for an extensive range of phenotypes. Genome-wide linkage scans for bone $e^{21,22}$ and eye disease ${ }^{23}$ have been reported previously for this cohort. Here we report the data from genome-wide linkage scans for obesity-related traits ( $n=2188$ individuals).

Expanded twin cohort for fine mapping In an attempt to improve the resolution of a linkage peak at $12 \mathrm{q} 24$ by fine mapping, we recruited and genotyped additional twins from the TwinsUK registry and the volunteer-based Sydney Longitudinal Study of Twins (Australia). These pairs were also Caucasian dizygous twin sisters between 18 and 80 years of age, and were subjected to the same data collection protocols described for the Initial Twin Cohort. This increased the number of pairs in the fine-mapping linkage analysis by approximately $14 \%$ to 1243 pairs.
Association cohort The analysis of single-nucleotide polymorphisms (SNPs) in positional candidate genes used dizygous twin pairs ( $n=1102$ individuals) selected from the extended twin cohort. Twins with the following conditions were excluded from the association study diabetes, thyroid disease, renal impairment, discordance for menopause status and those receiving lipid lowering medication, beta blockers, thiazide diuretics or exogenous estrogen. Pairs from the linkage studies were ranked for central fat mass concordance and discordance. Twins ( $n=551$ pairs) were selected sequentially from opposite ends of the trait distribution, maintaining a ratio of twin pairs who were discordant to those who were concordant of $5: 3 .^{24}$ Twins from the selected pedigrees were then genotyped for 45 SNPs in 26 candidate genes.

Phenotypes Measurement of obesity-related phenotypes $(g)$ : Total fat mass, total lean mass and total fat percentage were measured using a QDR-4500/w dual X-ray absorptiometer (Hologic Inc., Bedford MA, USA). ${ }^{25}$ Central fat mass (g) and central fat mass percentage were also derived from this analysis. $^{8}$ Height was measured without shoes using a Holtain stadiometer. Weight was measured when wearing light clothing and after removal of shoes, using a mechanical balance. Waist circumference was measured using a steel tape, halfway between the lower border of the ribs, and the iliac crest in a horizontal plane. Hip circumference was measured at the widest point over the buttocks. BMI and waist-to-hip ratio (WHR) were derived by computation. ${ }^{18}$

Ethics approval All subjects provided written informed consent and the institutional ethics committee of St Thomas' Hospital NHS Trust approved the experimental protocols.

\section{Genotyping}

Microsatellite marker-based genotyping ${ }^{26}$ was undertaken using standard ABI Prism ${ }^{\mathrm{TM}}$ methodologies (Applied Biosystems, Foster City, CA, USA) on DNA extracted from venous blood. Genome scans included the analysis of up to 737 markers on each individual as described previously. ${ }^{21}$ Zygosity was tested using AmpFLSTR DNA profiling kit (Applied Biosystem), with twins showing less than 100\% identity defined as dizygous.

Candidate genes were selected by prioritizing genes within the region based on biological properties that were consistent with a role in central adiposity. Considerations included: cDNA and EST sequences with tissue-specific expression (eg adipose, liver, brain and pancreas), potential contribution to obesity-related pathways and position. SNPs were selected based on multiple submissions to dbSNP, a unique validated position, availability of frequency data for Caucasian populations and minor allele frequency $>5 \%$, with a preference for coding SNPs. 
Table 1 Morphometric data for female dizygous twins

\begin{tabular}{lccr}
\hline & Initial twin cohort & Expanded twin cohort & Association cohort \\
\hline Age (years) & $47.0 \pm 11.5(2188)$ & $47.1 \pm 11.8(2486)$ & $45.5 \pm 12.5(1102)$ \\
Height $(\mathrm{m})$ & $1.63 \pm 0.06(2146)$ & $1.63 \pm 0.06(2444)$ & $1.63 \pm 0.06(1098)$ \\
Weight $(\mathrm{kg})$ & $66.2 \pm 12.5(2184)$ & $66.8 \pm 12.8(2482)$ & $66.8 \pm 13.4(1099)$ \\
Central fat mass $(\mathrm{g})$ & $1348 \pm 733(2048)$ & $1318 \pm 733(2486)$ & $1360 \pm 849(1102)$ \\
BMI $\left(\mathrm{kg} / \mathrm{m}^{2}\right)$ & $25.1 \pm 4.6(2146)$ & $25.2 \pm 4.7(2482)$ & $25.2 \pm 4.9(1098)$ \\
WHR & $0.77 \pm 0.06(2116)$ & $0.77 \pm 0.06(2420)$ & $0.77 \pm 0.06(1075)$ \\
\hline
\end{tabular}

Data are mean \pm SD (number of individuals).

Analysis of SNPs was analysed by nucleotide extension reaction on a MassArray MALDI-TOF mass spectrometer (Sequenom Inc., San Diego, CA, USA) or by Pyrosequencing on a PSQ96 (Pyrosequencing, Uppsala, Sweden), with reactions performed according to the manufacturer's instructions. Random duplicate genotyping was routinely undertaken throughout the study and indicated an error rate of $<1 \%$.

\section{Statistical analysis}

Nonparametric multipoint linkage (NPL) analyses were performed using the MAPMAKER/SIBS Ver 2.0 program. ${ }^{27}$ This method, based on the Wilcoxon rank-sum test, makes no assumptions about the distribution of phenotypic differences. The program also provides an estimate of the information content (IC; range $0-1$ ), or the proportion of maximum identity-by-descent (IBD) information that is extracted using the selected markers. ${ }^{27}$ Data from these NPL analyses were expressed as $Z$-scores and equivalent LOD scores. Marker loci map positions (NCBI Build 35) were determined from the Genethon linkage map as described previously. ${ }^{21}$ All phenotypes studied were analyzed as quantitative traits in univariate genome-wide scans. All twin pairs were from independent families. Significant and suggestive linkage was interpreted as corresponding to LOD scores of at least 3.3 and 1.9 , respectively. ${ }^{28}$ Simulated $P$-values were calculated on a genome-wide basis, with the phenotype data randomly reassigned to the genotypes for each pair in a minimum of 500 random reassignments, with $P<0.05$ considered as significant linkage. The approximate support interval for linkage was defined using the 1 LOD score interval. Genome-wide linkage scan data with a LOD $\geqslant 1$ and corresponding (within $5 \mathrm{cM}$ ) to a linkage for the same phenotype reported from other studies were considered supportive of previously established linkage.

A $\chi^{2}$ test was used to confirm that SNP data were in Hardy-Weinberg equilibrium. Single-point Monks-Kaplan association analysis, ${ }^{29}$ in the QPDTPHASE program, ${ }^{30}$ was used to test the relationship between SNPs and central fat mass. Haplotype analysis used the QPDTPHASE program, ${ }^{30}$ which calculates the expected covariance over a maximum-likelihood distribution of haplotype frequencies. Analysis of anthropometric data was performed using Statistica (Statsoft Inc. 1998, Ver 6.0, Tulsa, OK, USA).

\section{Results}

The mean age of the twin cohort was $47.0 \pm 11.5$ years (range 18.4-79.1). These women had a mean BMI on the upper boundary of normal BMI $\left(25.1 \pm 4.6 \mathrm{~kg} / \mathrm{m}^{2}\right.$, range 13.9-52.4; Table 1), consistent with a general trend towards overweight in the UK population. Overall data for obesity-related phenotypes were consistent with agematched singleton population-based samples. ${ }^{31}$

NPL analyses of obesity-related traits provided support for previously established linkage of central fat mass to 12q24 (141 cM; Figure 1d) with LOD 2.2, and for BMI to $8 q 11$ ( $67 \mathrm{cM}$; Figure 1f) with LOD 1.3. There were novel suggestive linkages for total fat percentage, maximal at 6q12-13 (LOD 2.4; 81 cM; Figure 1c), and total lean mass at 2q37 (LOD 2.4; $246 \mathrm{cM}$; Figure 1a). An additional novel linkage for central fat mass at $1 \mathrm{p} 36$ (LOD 2.1; $48 \mathrm{cM}$; Figure 1d) was also observed. Empirical $P$-values generated using a permutation approach for these linkages did not achieve conventional genome-wide significance. The approximate support interval for the central fat masschromosome $12 \mathrm{q}$ linkage extended between 130 and $165 \mathrm{cM}$ (Figure 2; flanked by markers D12S79 and D12S1723). The approximate support interval for the chromosome 6q linkage for percentage total fat was $35 \mathrm{cM}$, and extended between 70 and $105 \mathrm{cM}$ (flanked by D6S426 and D6S262).

As the twins in this cohort represent a distribution of individuals from lean to obese (Table 1), we also performed an analysis of data for lean body mass to localize genes that control leanness. Total lean mass showed suggestive linkage to 2q37 (LOD 2.4; Figure 1a), with an approximate support interval of $22 \mathrm{cM}$, which extended between 235 and $257 \mathrm{cM}$ (flanked by D2S2308 and D2S338). Additional linkage for total lean mass with $\mathrm{LOD}>2$ was to $14 \mathrm{q} 11$ (Figure 1a). It should be noted that total lean mass, unadjusted for total mass, is not necessarily indicative of true leanness (i.e. obese subjects can have a high lean mass). Furthermore, since total lean mass and total fat mass change substantially and in a nonparallel way over the age 

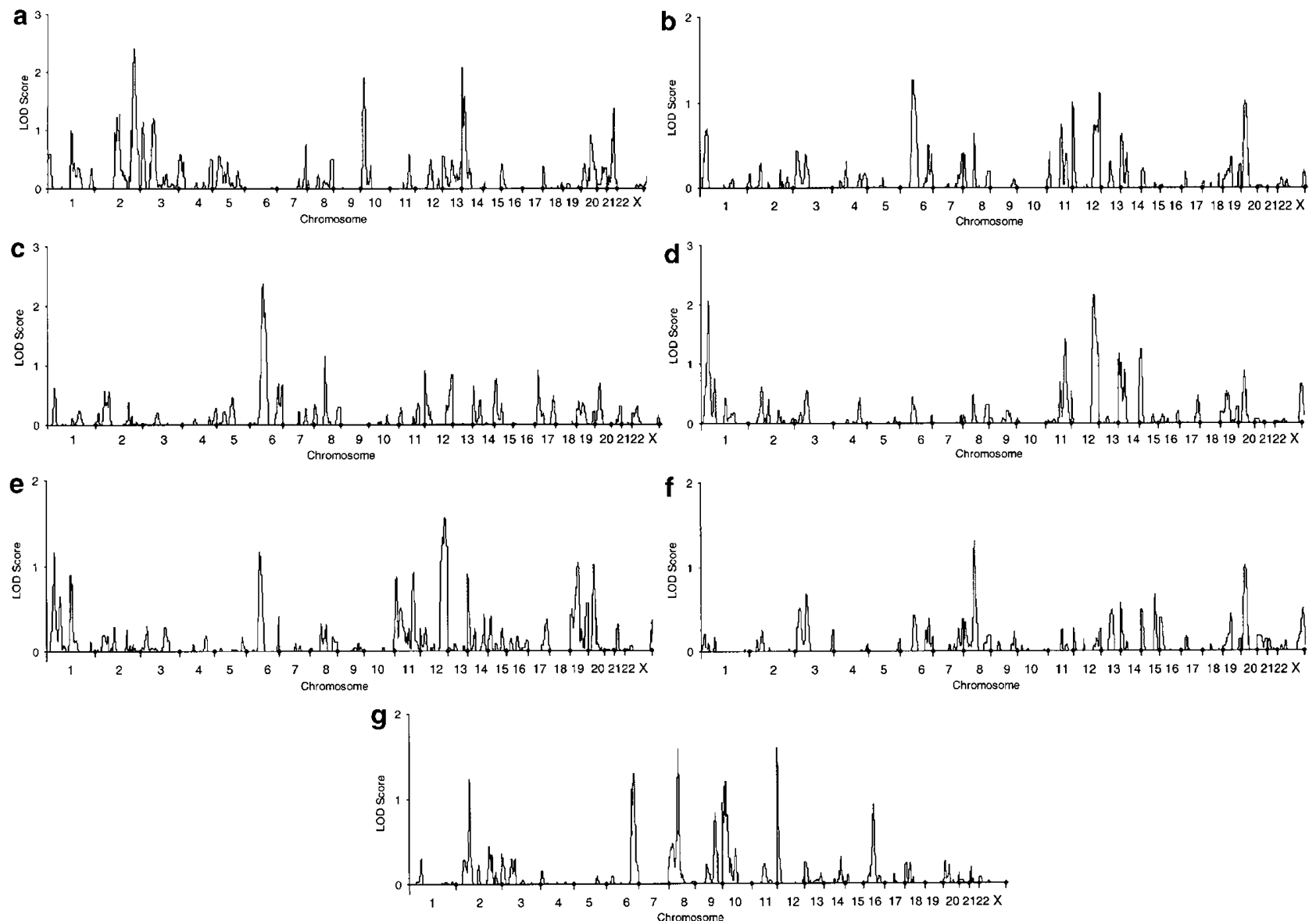

Figure 1 Genome-wide scans for QTL that regulate (a) total lean mass, (b) total fat mass, (c) total fat percentage, (d) central fat mass, (e) central fat mass percentage, (f) body mass index and (g) WHR in 1094 twin pairs. Equivalent LOD scores (LOD) were calculated from the NPL Z-scores output by MAPMAKER/SIBS, using the relationship $Z^{2} / 4.6052=$ LOD.

range of the subjects studied, these data must be interpreted cautiously. Additional weaker evidence of linkage $(L O D \geqslant 1)$ in the cohort was seen to nine other genomic regions with obesity-related traits (Table 2 ).

The traits we analyzed could be expected to have some level of correlation. Genome-wide linkage scans for the most strongly correlated variables: total fat and total fat percentage $(r=0.93, n=2030)$ and central fat mass and central fat mass percentage $(r=0.94, n=2044)$, do show substantial similarity. Nevertheless, for total fat and total fat percentage, the magnitude of LOD scores for comparable linkage peaks on chromosome 6 at $81-83 \mathrm{cM}$ varied by almost two-fold (Figure $1 \mathrm{~b}$ and c). Similarly, with central fat mass and central fat percentage, the magnitude of the LOD scores on chromosome 1 at $48 \mathrm{cM}$ differed greatly (Figure $1 \mathrm{~d}$ and e). While there was a strong phenotypic correlation between total fat mass and central fat mass ( $r=0.89, n=2033$ ), the genome scans for these variables showed less concordance and may still be potentially relevant to general overweight and obesity, or regional deposition of fat on the trunk, respectively. Data for other obesity-related traits such as BMI and WHR showed lesser correlation with central fat mass (ie $r=0.81, n=2008$ and $r=0.494, n=1986$, respectively) and result in correspondingly unique linkage data (Figure if and g).

As the $12 \mathrm{q}$ linkage for central fat mass (Table 2) was among the largest we defined and supported a previous report, ${ }^{32}$ we focused additional efforts on that region. NPL analysis of data on the base set of markers for the expanded cohort of 1243 twin pairs reinforced and enhanced the level of linkage on chromosome $12 \mathrm{q} 24$ for central fat mass (LOD 2.9) and central fat mass percentage (LOD 2.1). Due to resource constraints, fine mapping for the 12q24 linkage was undertaken with only seven additional microsatellite markers (Figure 2). As the support interval was relatively large and asymmetric, we placed fine mapping markers towards the q-telomere to increase the IC in this region. NPL analysis of the fine-mapping data for central fat in the expanded cohort yielded a LOD of $2.6(143 \mathrm{cM})$. This analysis increased confidence in the linkage results by removing reliance on only a few microsatellite markers and narrowed the support interval to $22 \mathrm{cM}$ (Figure 2). The 


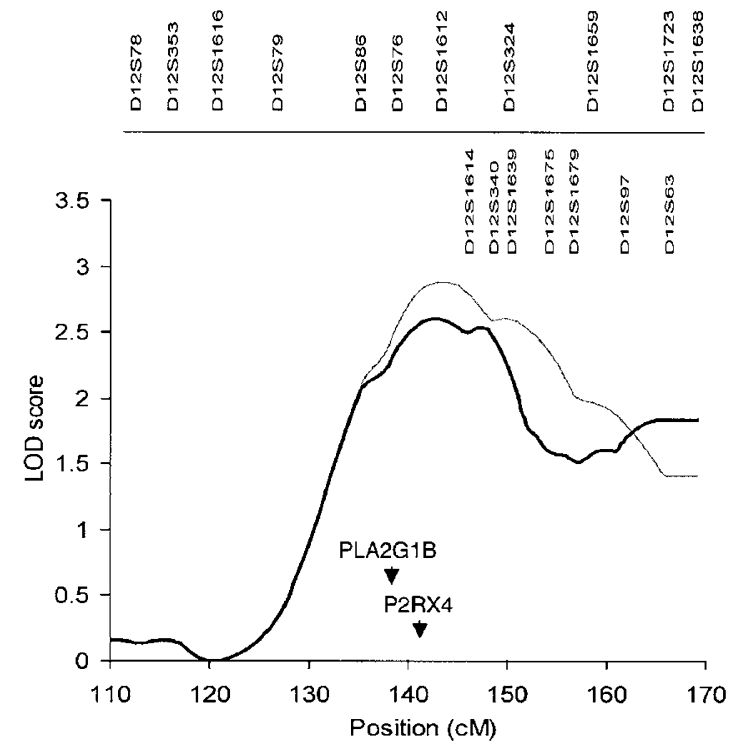

Figure 2 Maximum NPL for the central fat mass phenotype on chromosome 12q in 1243 twin pairs. Linkage analysis results are presented for the expanded twin cohort with the core markers used in the initial scan (-) and after genotyping of additional microsatellite markers (-). The top row of marker names are the core markers, finemapping markers are defined on the second row. mean IC in the interval rose from 0.61 to 0.72 as a result of the fine mapping.

In all, 26 genes, implicated as potential candidates in the 12 q24 region, were identified from public domain resources. Analysis of data for 45 SNPs in these genes (Table 3) provided evidence of association with central fat in women, for two genes (Figure 3 ). The lowest $P$-value with central fat mass was with SNP rs5637 in exon 3 of secretory phospholipase $\mathrm{A}_{2}$ precursor gene (PLA2G1B; $P=0.0067$, $n=1066)$. Allele frequencies for rs5637 were 0.853 for G-allele and 0.147 for A-allele, with the A-allele carriers having lower mean central fat mass (Table 4, Figure 3a). The SNP rs1179387 in intron 3 of PLA2G1B also showed association with central fat mass $(P=0.0069, n=1059$, allele frequencies: $A=0.852, C=0.148)$. Lewontin's $D^{\prime}$ for these two SNPs was 0.99, illustrating that the two SNPs are in strong linkage disequilibrium. Haplotype analysis provided maximum evidence of association with a four-SNP haplotype encompassing rs2009391, rs5634, rs5637 and rs1179387 (Table 5, $P=0.0026$, T-T-A-G, frequency: 0.14).

One SNP within the ligand-gated cation channel gene P2RX4 (Figure 3b) also showed nominal association with central fat mass in the study cohort: rs1182945 in intron $2(P=0.017, n=1055$, allele frequencies: $A=0.668$,

Table 2 Genomic location of maximum NPL for obesity-related phenotypes in unselected female twins with LOD scores above 1

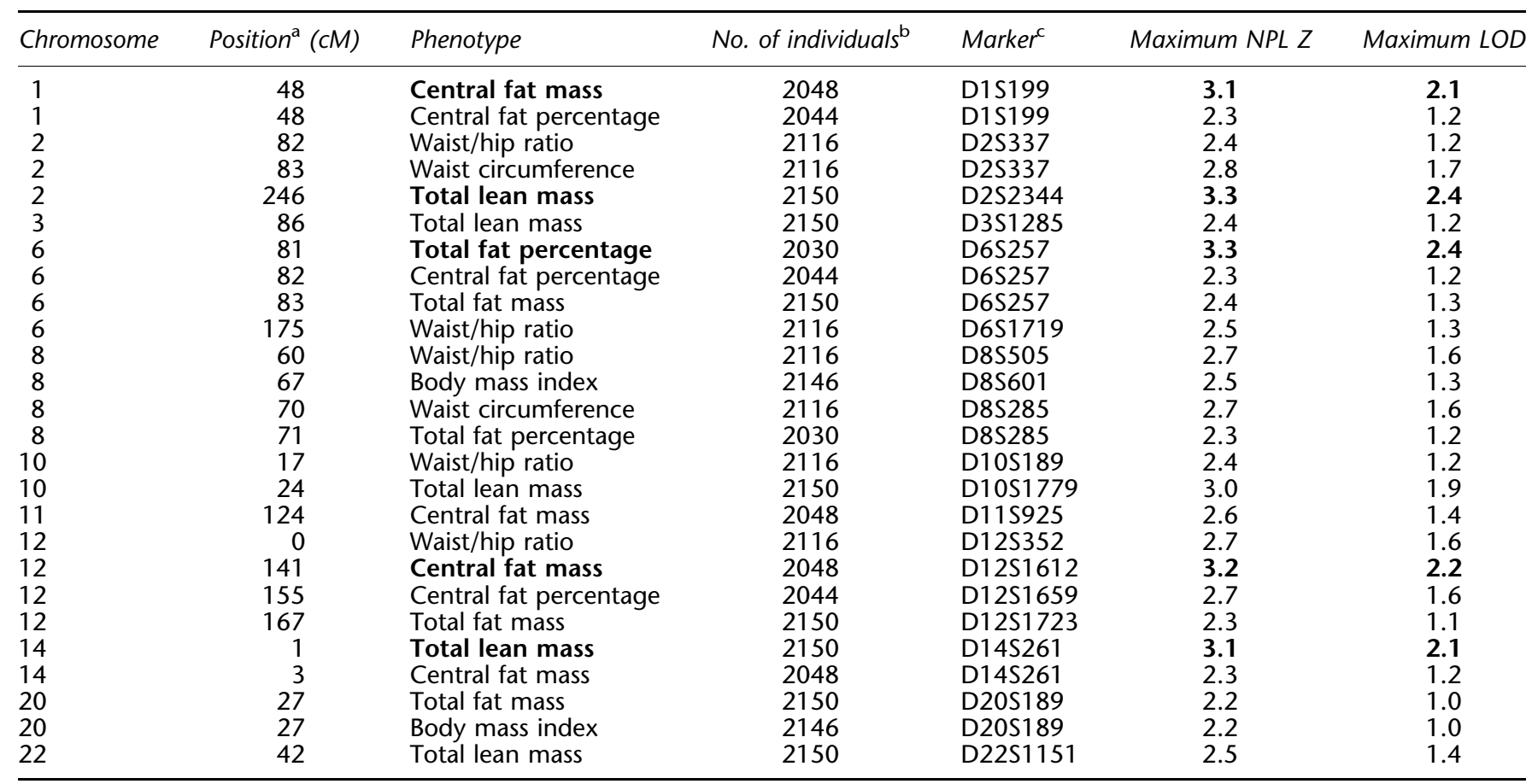

${ }^{a}$ Distance of the linkage peak from the $p$ telomere.

${ }^{b}$ Number of individuals differs depending on the availability of phenotype data.

${ }^{\mathrm{c}}$ Closest microsatellite marker genotyped to the apex of the linkage peak. 
Table 3 Positional candidate genes studied for association with central fat mass and P-values for Monks-Kaplan association test with central fat mass in the association cohort

\begin{tabular}{|c|c|c|c|}
\hline Gene & $\begin{array}{c}d b S N P \text { reference } \\
\text { number }(r s)\end{array}$ & Position Chr12 & P-value \\
\hline \multirow[t]{3}{*}{ ACACB } & 34269 & 108060225 & 0.4 \\
\hline & 34280 & 108066955 & 0.9 \\
\hline & $1016331^{H}$ & 108087016 & 0.6 \\
\hline TBX5 & 1895584 & 113269255 & 0.7 \\
\hline TBX3 & 539709 & 113582034 & 0.6 \\
\hline \multirow[t]{3}{*}{ NOS1 } & 816358 & 116160028 & 0.8 \\
\hline & 733334 & 116195409 & 0.14 \\
\hline & 567581 & 116220232 & 0.5 \\
\hline PBP & 904662 & 117044553 & 0.9 \\
\hline HSPB8 & 1966544 & 118088842 & 0.2 \\
\hline \multirow[t]{2}{*}{ PRKAB1 } & 278145 & 118574819 & 0.4 \\
\hline & $278152^{H}$ & 118579999 & 0.4 \\
\hline \multirow[t]{4}{*}{ PLA2G1B } & $1179387^{H}$ & 119223631 & 0.0069 \\
\hline & 5637 & 119225485 & 0.0067 \\
\hline & $5634^{H}$ & 119225557 & 0.6 \\
\hline & $2009391^{H}$ & 119228763 & 0.4 \\
\hline COX6A1 & $968474^{H}$ & 119341250 & 1 \\
\hline \multirow[t]{2}{*}{ ACADS } & 575437 & 119636151 & 0.5 \\
\hline & $9204^{H}$ & 119640498 & 0.4 \\
\hline \multirow[t]{2}{*}{ TCF1 } & $1169288^{H}$ & 119879370 & 0.9 \\
\hline & $1169302^{H}$ & 119895022 & 0.19 \\
\hline \multirow{3}{*}{$\begin{array}{l}\text { P2RX7 } \\
\text { P2RX4 }\end{array}$} & 208307 & 120066576 & 0.5 \\
\hline & 1182945 & 120118690 & 0.017 \\
\hline & $1151880^{H}$ & 120130371 & 0.4 \\
\hline PSMD9 & 483416 & 120800833 & 0.7 \\
\hline DIABLO & $12870^{H}$ & 121217700 & 0.7 \\
\hline FLJ13491 & 883562 & 121985599 & 0.3 \\
\hline PITPNM2 & 1984658 & 122008306 & 0.5 \\
\hline CDK2AP1 & 6633 & 122270689 & 0.5 \\
\hline GTF2H3 & 1051793 & 122669275 & 1 \\
\hline ATP6VOA2 & $1040156^{H}$ & 122748244 & 0.3 \\
\hline FLJ22471 & $1882491^{H}$ & 122979115 & 0.2 \\
\hline \multirow[t]{6}{*}{ NCOR2 } & $8546^{H}$ & 123333907 & 0.16 \\
\hline & 1271309 & 123345585 & 0.3 \\
\hline & 1244085 & 123354135 & 0.09 \\
\hline & $906302^{H}$ & 123375337 & 0.5 \\
\hline & 1244053 & 123415092 & 0.9 \\
\hline & 872224 & 123504713 & 0.7 \\
\hline \multirow[t]{2}{*}{ SCARB1 } & 1814575 & 123804271 & 0.8 \\
\hline & 5888 & 123809628 & 0.17 \\
\hline AACS & 914610 & 124080119 & 0.5 \\
\hline \multirow{2}{*}{ FLJ31978 } & 527824 & 127880953 & 0.6 \\
\hline & $470576^{H}$ & 127942190 & 0.07 \\
\hline \multirow[t]{2}{*}{ SFRS8 } & 897646 & 130790890 & 1 \\
\hline & 755437 & 130926532 & 0.7 \\
\hline
\end{tabular}

${ }^{H}$ SNP data present in HapMap.

$T=0.332$ ). Carriage of the A-allele in this SNP was associated with higher mean central fat mass (Table 4), although it should be noted that, due to the nature of the Monks-Kaplan association test, the $P$-value is based on within-family association and not a direct test of the genotypic means. Pairwise $D^{\prime}$ values were calculated for the SNPs genotyped in PLA2G1B and P2RX4, and, despite the relatively close physical mapping of the two genes, no evidence of linkage disequilibrium was found between the genes.

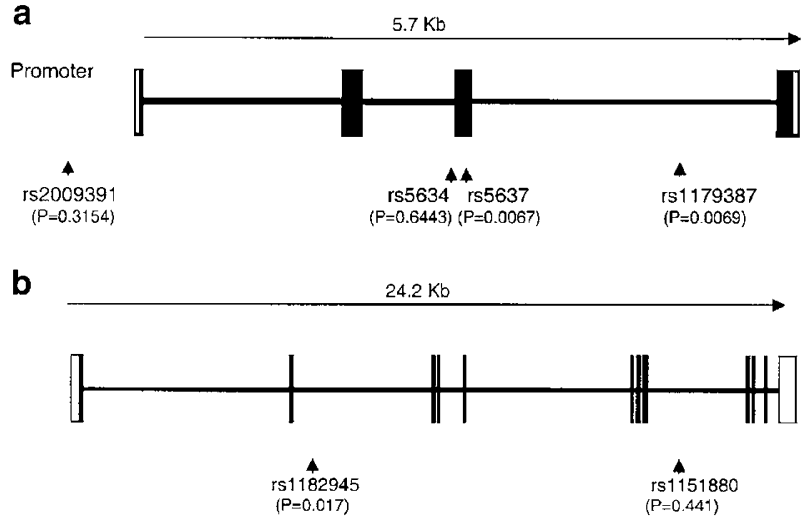

Figure 3 Position of SNPs (designated by reference SNP number) studied in (a) PLA2G1B and (b) P2RX4. P-values for Monks Kaplan association test with central fat mass are shown below each SNP. $\square=$ untranslated region, $\mathbf{\square}=$ exon

Table 4 Genotype frequencies and central fat mass data for individuals grouped by genotype for two SNPs in positional candidate genes on chromosome $12 q 24$

\begin{tabular}{llcr}
\hline Gene (SNP) & Genotype & Mean \pm SD $(g)$ & $n$ \\
\hline PLA2G1B (rs5637) & A/A & $1256 \pm 802^{\mathrm{a}}$ & 28 \\
& A/G & $1267 \pm 784$ & 272 \\
& G/G & $1387 \pm 869$ & 766 \\
P2RX4 (rs1182945) & T/T & $1341 \pm 847$ & 116 \\
& A/T & $1342 \pm 846$ & 456 \\
& A/A & $1363 \pm 841^{\mathrm{b}}$ & 483 \\
\hline
\end{tabular}

Significant difference by Monks-Kaplan single-point association test. ${ }^{\text {a }} P=0.0067$

${ }^{\mathrm{b}} P=0.017$.

\section{Discussion}

This study of female twins provides evidence of support for previously established linkage of central fat mass to $12 q^{32}$ and for BMI to $8 \mathrm{q} \cdot{ }^{33,34}$ The use of a positional candidate approach to identify QTLs in the region resulted in the detection of statistically significant associations between SNPs in the PLA2G1B and P2RX4 genes and central fat mass in women.

The deposition of fat on the trunk and waist is a predominant risk factor for disorders such as metabolic syndrome, non-insulin-dependant diabetes and cardiovascular disease. ${ }^{8,35}$ The metabolic properties of visceral and subcutaneous adipocytes are also known to differ, with visceral cells being more sensitive to lipolytic stimuli and less sensitive to the inhibitory effect on lipolysis by insulin. ${ }^{35-37}$ Furthermore, the prevalence of abdominal obesity and its relationship to generalized obesity differs with ethnic group, gender ${ }^{38,39}$ and menopause. ${ }^{8}$ We found support for the previously established linkage observed in the Québec Family Study, ${ }^{32}$ which used computed tomography (CT) to estimate fat mass in 521 subjects. Our study 
Table 5 Effect of the PLA2G1B haplotypes on central fat mass in female dizygous twins

\begin{tabular}{|c|c|c|c|c|}
\hline Haplotypes $^{\mathrm{a}}$ & $G-T-G-T$ & $T-T-G-T$ & $T-T-A-G$ & $G-C-G-T$ \\
\hline $\begin{array}{l}\text { Haplotype frequency }(\%)^{b} \\
\text { Central fat mass }(g)^{c}\end{array}$ & $\begin{array}{c}51 \\
1362 \pm 30\end{array}$ & $\begin{array}{c}30 \\
1341 \pm 39\end{array}$ & $\begin{array}{c}14 \\
1274 \pm 57^{d}\end{array}$ & $\begin{array}{c}4 \\
1464 \pm 107\end{array}$ \\
\hline
\end{tabular}

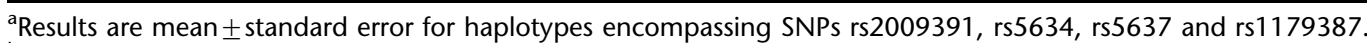

${ }^{\mathrm{b}}$ Rare haplotypes (frequency less than $3 \%$ ) not included.

${ }^{\mathrm{C}} P=0.03$ overall association with central fat mass.

${ }^{\mathrm{d}} P=0.0026$ for individual haplotype compared to all others.

included nearly twice as many subjects, but CT was not used. CT is widely regarded as the method of choice for precise measurement of abdominal subcutaneous and visceral fat, but involves considerable radiation. Absorptiometry does not provide the same detail, but has been shown to be a reproducible technique for assessment of obesity-related traits, including central fat mass. ${ }^{8,25}$ In another study, linkage for obesity was also found to 12 q24 in patients on antipsychotic medication. ${ }^{40}$ Recently, Li et al $^{41}$ have shown significant linkage for obesity-related traits: percentage fat (LOD 3.79; $P=0.00001$ ), BMI (LOD 2.98; $P=0.0001$ ) and waist circumference (LOD 2.86; $P=0.00014)$ to chromosome $12 \mathrm{q} 23-24$. Their fine-mapping data localized linkages for those traits to $116-125 \mathrm{cM}$ on chromosome 12. There does not appear to be an overlap between the -1 LOD approximate support interval reported in that paper and our data; however, the proximity of the linkages is intriguing. Studies of non-insulin-dependant diabetes in humans ${ }^{42-44}$ have also highlighted this genomic region as important, raising the possibility that QTLs in the region may be implicated in several disease states. We also found linkage of central fat mass to chromosome 1p36 (LOD 2.1). Another study ${ }^{33}$ found strong evidence of linkage on chromosome $1(45-48 \mathrm{cM})$ for BMI; however, they did not consider measures of central obesity. In contrast, we did not find linkage of BMI to this region of chromosome 1 and so, despite the recognition of potential concordance of the linkage peaks, we remain cautious about the possible overinterpretation of this data.

Our data showed evidence of support for linkage of BMI to chromosome $8 \mathrm{p} 12-\mathrm{q} 22.1$. This linkage had previously been reported in 10 large families of Mexican Americans from a fine-mapping study of 8 p12-q22.1 (LOD 3.21 at $63 \mathrm{cM}){ }^{34}$ which is within $4 \mathrm{cM}$ of the linkage for BMI that we report. Participants in that study had an increased BMI $\left(29.7 \mathrm{~kg} / \mathrm{m}^{2}\right)$ compared to our subjects, but were not morbidly obese. Linkage of BMI to $8 \mathrm{q} 12$ has also been observed in a large cohort of white children and young adults from the Rochester Family Heart study. ${ }^{33}$ That study also provided evidence of linkage for BMI to 20p12-13, a finding supported by our data. Other presently unsupported linkage areas from this genome scan, particularly those with $\operatorname{LOD}>2$, may aid in prioritizing regions for further study among numerous linked regions reported to date. ${ }^{13}$ In a recent review, Perusse et al ${ }^{13}$ reported that there are at least 204 QTLs for obesity-related phenotypes from 50 genome-wide linkage scans, with 38 genomic regions reported as being supported by data from at least two studies. Care must be taken when interpreting this data and further studies may be required on the genomic regions and positional candidates identified in this study to clarify their relevance.

Our work on fine mapping in the expanded cohort helped to confirm suggestive linkage of central fat mass to 12q24, narrow the support interval (LOD 2.6; $143 \mathrm{cM}$ ) and facilitate a study of positional candidates in the region. It is well known that the principle candidate gene within a linkage region, identified on the basis of known function, may not be the source of the observed linkage, so we studied SNP in a substantial number of the potential candidate genes in the region. There are over 120 genes within the support interval, but due to resource limitations we chose only 26 genes for study and attempted to expeditiously sample possible QTLs. The number of pairs we used in the association study was defined by power calculations which suggested that a QTL accounting for $10 \%$ of the phenotypic variance, with a $D^{\prime}$ of 0.5 between the marker and the QTL, no dominance and a minor allele frequency of 0.2 , would require $552 \mathrm{sib}$ pairs to achieve $80 \%$ power with an $\alpha=0.001$. In comparison, for an effect size of $5 \%$, the number of pairs required was estimated as 1112. Our data is useful in emphasizing both the size of cohorts needed for such studies and the scale of investigation that may generally be required to uncover QTLs in positional candidate studies.

We found evidence of association between SNPs in the PLA2G1B gene and central fat mass. Phospholipases (PLA2) are small lipolytic enzymes that release fatty acids from the second carbon group of glycerol. The best known of these are the digestive enzymes secreted as zymogens by the pancreas. $P L A 2 G 1 B$ is the pancreatic form and is thought to hydrolyse phospholipids to increase free fatty acids and lysolipids. Huggins et al $^{45}$ demonstrated previously that $\mathrm{PLA}_{2}^{-1-}$ mice fed a high-fat diet were resistant to diet-induced obesity. In the light of that result, there may be a plausible role for genetic variation in the PLA2G1B gene in protection against diet-induced obesity. The SNP rs5637, which showed the strongest association with central fat mass, results in synonymous coding for serine. In comparison, rs1179387 is in intron 3 of the PLA2G1B gene, suggesting 
that both SNPS may be in linkage disequilibrium with a functional variant(s), yet to be determined.

The product of the P2RX4 gene belongs to the family of purinoceptors for ATP. This receptor functions as a cell surface ligand-gated ion channel with high calcium permeability. Expression of the gene in the rat has shown P2RX4 levels to be high in the brain, suggesting a possible role in appetite control. In humans, the gene is expressed at high levels in the pancreas, stomach and small intestine, and at lower levels in the brain. Interestingly, ethanol has been found to inhibit the opening of channels regulated by P2RX4 in Xenopus oocytes. ${ }^{46}$ As ethanol is known to effect the regulation of leptin, ${ }^{47}$ reduced levels of which stimulate appetite, there is a possibility that $P 2 R X 4$ could also exert effects on fat metabolism via a link with ethanol or leptin. SNP rs1182945, which showed potential association with central fat mass, is in intron 2 of the P2RX4 gene and so it seems likely that a functional change, in linkage disequilibrium with this SNP, is responsible for the observed effect on central fat mass.

Other studies of candidate genes in the 12 q24 region ${ }^{48,49}$ have found association of BMI with genetic variation in the SCARB1 gene, but we did not find any association between the two SNPs we studied and either central fat mass or BMI. Mutations in the TCF1 gene are thought to be responsible for Mature Onset Diabetes of the Young in some patients (MODY3) ${ }^{50-53}$ However, we found no association between the SNP in this gene and central fat mass in female twins.

We performed a substantial number of statistical tests in the association study and present these data without correcting for multiple testing. Application of the conservative Bonferroni correction - assuming the SNPs are uncorrelated - would be one method to account for this issue, but this is considered too conservative by most researchers. ${ }^{54}$ Presentation of the data without correction may be important for later meta-analysis. We cautiously advocate only that SNP in PLA2G1B and P2RX4 show possible association, which will need to be verified by replication. We also performed only a superficial sampling of SNPs in potentially relevant genes. We did this in an attempt to gain insight and to aid in prioritization of genes and SNPs in the region for further study. This approach has yielded two potentially relevant associations, but much work remains to be performed in the region.

In summary, despite early disappointment with the positional cloning approach for the discovery of complex disease genes, recent successes, the current support for established linkages and the use of selected intermediate phenotypes provide considerable room for optimism. If these results for $P L A 2 G 1 B$ and $P 2 R X 4$ can be verified through replication or functional analysis, the recognition that these polymorphisms are specifically associated with accumulation of abdominal fat could be a major contribution to combating the development of disorders such as the metabolic syndrome and other related cardiovascular conditions.

\section{Acknowledgements}

We thank Lars Berglund, Mathias Chiano and the staff of SequenomGemini Ltd (UK and Sweden), who contributed to genotyping and statistical analysis. TwinsUK receives funding from the Wellcome Trust, the CDRF and the Biomed EU GenomEUtwin and EuroClot project (Ref: LSHM-CT-2004-005268, QLK2-CT-2002-01254) programs. Lastly we would like to thank the twins themselves.

\section{References}

1 Caterson ID, Gill TP: Obesity: epidemiology and possible prevention. Best Pract Res Clin Endocrinol Metab 2002; 16: 595-610.

2 Greenfield JR, Samaras K, Jenkins AB et al: Obesity is an important determinant of baseline serum C-reactive protein concentration in monozygotic twins, independent of genetic influences. Circulation 2004; 109: 3022-3028.

3 Toni R, Malaguti A, Castorina S, Roti E, Lechan RM: New paradigms in neuroendocrinology: relationships between obesity, systemic inflammation and the neuroendocrine system. J Endocrinol Invest 2004; 27: 182-186.

4 Lau DC, Dhillon B, Yan H, Szmitko PE, Verma S: Adipokines: molecular links between obesity and atherosclerosis. Am J Physiol Heart Circ Physiol 2005; 288: H2031-H2041.

5 Flier JS, Foster DW: Eating disorders: obesity, anorexia nervosa and bulimia nervosa; in Wilson JD, Foster DW, Kronenberg HM, Larsen PR (eds): Williams textbook of endocrinology. Philadelphia, USA: WB Saunders Company, 1998, pp 1061-1097.

6 Rose KM, Newman B, Mayer-Davis EJ, Selby JV: Genetic and behavioral determinants of waist-hip ratio and waist circumference in women twins. Obes Res 1998; 6: 383-392.

7 Austin MA, Friedlander Y, Newman B, Edwards K, Mayer-Davis EJ, King MC: Genetic influences on changes in body mass index: a longitudinal analysis of women twins. Obes Res 1997; 5: 326-331.

8 Samaras K, Spector TD, Nguyen TV, Baan K, Campbell LV, Kelly PJ: Independent genetic factors determine the amount and distribution of fat in women after the menopause. J Clin Endocrinol Metab 1997; 82: 781-785.

9 Schousboe K, Visscher PM, Erbas B et al: Twin study of genetic and environmental influences on adult body size, shape, and composition. Int J Obes Relat Metab Disord 2004; 28: 39-48.

10 Juo SH, Lin HF, Rundek $\mathrm{T}$ et al: Genetic and environmental contributions to carotid intima-media thickness and obesity phenotypes in the Northern Manhattan Family Study. Stroke 2004; 35: 2243-2247.

11 Rice T, Perusse L, Bouchard C, Rao DC: Familial aggregation of body mass index and subcutaneous fat measures in the longitudinal Quebec family study. Genet Epidemiol 1999; 16: 316-334.

12 Pausova Z, Gossard F, Gaudet D et al: Heritability estimates of obesity measures in siblings with and without hypertension. Hypertension 2001; 38: 41-47.

13 Perusse L, Rankinen T, Zuberi A et al: The human obesity gene map: the 2004 update. Obes Res 2005; 13: 381-490.

14 Durand E, Boutin P, Meyre D et al: Polymorphisms in the amino acid transporter solute carrier family 6 (neurotransmitter transporter) member 14 gene contribute to polygenic obesity in French Caucasians. Diabetes 2004; 53: 2483-2486.

15 Suviolahti E, Oksanen LJ, Ohman M et al: The SLC6A14 gene shows evidence of association with obesity. J Clin Invest 2003; 112: $1762-1772$.

16 Ohman M, Oksanen L, Kaprio J et al: Genome-wide scan of obesity in Finnish sibpairs reveals linkage to chromosome Xq24. J Clin Endocrinol Metab 2000; 85: 3183-3190.

17 Moller DE, Kaufman KD: Metabolic syndrome: a clinical and molecular perspective. Annu Rev Med 2005; 56: 45-62.

18 Carey DG, Jenkins AB, Campbell LV, Freund J, Chisholm DJ: Abdominal fat and insulin resistance in normal and overweight women: direct measurements reveal a strong relationship in 
subjects at both low and high risk of NIDDM. Diabetes 1996; 45: 633-638.

19 Carr MC: The emergence of the metabolic syndrome with menopause. J Clin Endocrinol Metab 2003; 88: 2404-2411.

20 Poirier P, Despres JP: Exercise in weight management of obesity. Cardiol Clin 2001; 19: 459-470.

21 Wilson SG, Reed PW, Bansal A et al: Comparison of genome screens for two independent cohorts provides replication of suggestive linkage of bone mineral density to $3 p 21$ and 1 p36. Am J Hum Genet 2003; 72: 144-155.

22 Wilson SG, Reed PW, Andrew T et al: A genome-screen of a large twin cohort reveals linkage for quantitative ultrasound of the calcaneus to 2q33-37 and 4q12-21. J Bone Miner Res 2004; 19: 270-277.

23 Hammond CJ, Andrew T, Mak YT, Spector TD: A susceptibility locus for myopia in the normal population is linked to the PAX6 gene region on chromosome 11: a genomewide scan of dizygotic twins. Am J Hum Genet 2004; 75: 294-304.

24 Dolan CV, Boomsma DI: Optimal selection of sib pairs from random samples for linkage analysis of a QTL using the EDAC test. Behav Genet 1998; 28: 197-206.

25 Albanese CV, Diessel E, Genant HK: Clinical applications of body composition measurements using DXA. J Clin Densitom 2003; 6: $75-85$.

26 Reed PW, Davies JL, Copeman JB et al: Chromosome-specific microsatellite sets for fluorescence-based, semi-automated genome mapping. Nat Genet 1994; 7: 390-395.

27 Kruglyak L, Lander ES: Complete multipoint sib-pair analysis of qualitative and quantitative traits. Am J Hum Genet 1995; 57: $439-454$.

28 Lander E, Kruglyak L: Genetic dissection of complex traits: guidelines for interpreting and reporting linkage results. Nat Genet 1995; 11: 241-247.

29 Monks SA, Kaplan NL: Removing the sampling restrictions from family-based tests of association for a quantitative-trait locus. Am J Hum Genet 2000; 66: 576-592.

30 Dudbridge F: Pedigree disequilibrium tests for multilocus haplotypes. Genet Epidemiol 2003; 25: 115-121.

31 Andrew T, Hart DJ, Snieder H, de Lange M, Spector TD, MacGregor AJ: Are twins and singletons comparable? A study of disease-related and lifestyle characteristics in adult women. Twin Res 2001; 4: 464-477.

32 Perusse L, Rice T, Chagnon YC et al: A genome-wide scan for abdominal fat assessed by computed tomography in the Quebec Family Study. Diabetes 2001; 50: 614-621.

33 Gorlova OY, Amos CI, Wang NW, Shete S, Turner ST, Boerwinkle E: Genetic linkage and imprinting effects on body mass index in children and young adults. Eur J Hum Genet 2003; 11: 425-432.

34 Mitchell BD, Cole SA, Comuzzie AG et al: A quantitative trait locus influencing BMI maps to the region of the beta-3 adrenergic receptor. Diabetes 1999; 48: 1863-1867.

35 Must A, Spadano J, Coakley EH, Field AE, Colditz G, Dietz WH: The disease burden associated with overweight and obesity. JAMA 1999; 282: 1523-1529.

36 Williams CM: Lipid metabolism in women. Proc Nutr Soc 2004; 63: $153-160$.

37 Wajchenberg BL, Giannella-Neto D, da Silva ME, Santos RF: Depot-specific hormonal characteristics of subcutaneous and visceral adipose tissue and their relation to the metabolic syndrome. Horm Metab Res 2002; 34: 616-621.

38 McKeigue PM, Shah B, Marmot MG: Relation of central obesity and insulin resistance with high diabetes prevalence and cardiovascular risk in South Asians. Lancet 1991; 337: 382-386.

39 McKeigue PM, Pierpoint T, Ferrie JE, Marmot MG: Relationship of glucose intolerance and hyperinsulinaemia to body fat pattern in south Asians and Europeans. Diabetologia 1992; 35: 785-791.

40 Chagnon YC, Merette C, Bouchard RH, Emond C, Roy MA, Maziade M: A genome wide linkage study of obesity as secondary effect of antipsychotics in multigenerational families of eastern Quebec affected by psychoses. Mol Psychiatry 2004; 9: 1067-1074.

$41 \mathrm{Li}$ WD, Dong C, Li D, Zhao H, Price RA: An obesity-related locus in chromosome region 12q23-24. Diabetes 2004; 53: 812-820.

42 Shaw JT, Lovelock PK, Kesting JB et al: Novel susceptibility gene for late-onset NIDDM is localized to human chromosome 12q. Diabetes 1998; 47: 1793-1796.

43 Lindgren CM, Mahtani MM, Widen E et al: Genomewide search for type 2 diabetes mellitus susceptibility loci in Finnish families: the Botnia study. Am J Hum Genet 2002; 70: 509-516.

44 Demenais F, Kanninen T, Lindgren CM et al: A meta-analysis of four European genome screens (GIFT Consortium) shows evidence for a novel region on chromosome 17p11.2-q22 linked to type 2 diabetes. Hum Mol Genet 2003; 12: 1865-1873.

45 Huggins KW, Boileau AC, Hui DY: Protection against diet-induced obesity and obesity-related insulin resistance in Group 1B PLA2-deficient mice. Am J Physiol Endocrinol Metab 2002; 283: E994-E1001.

46 Xiong K, Li C, Weight FF: Inhibition by ethanol of rat P2X(4) receptors expressed in Xenopus oocytes. Br J Pharmacol 2000; 130: $1394-1398$.

47 Kiefer F, Jahn H, Wolf K, Kampf P, Knaudt K, Wiedemann K: Free-choice alcohol consumption in mice after application of the appetite regulating peptide leptin. Alcohol Clin Exp Res 2001; 25: 787-789.

48 Acton S, Osgood D, Donoghue M et al: Association of polymorphisms at the SR-BI gene locus with plasma lipid levels and body mass index in a white population. Arterioscler Thromb Vasc Biol 1999; 19: 1734-1743.

49 Koumanis DJ, Christou NV, Wang XL, Gilfix BM: Pilot study examining the frequency of several gene polymorphisms in a morbidly obese population. Obes Surg 2002; 12: 759-764.

50 Menzel S, Yamagata K, Trabb JB et al: Localization of MODY3 to a 5-cM region of human chromosome 12. Diabetes 1995; 44: $1408-1413$

51 Yamagata K, Oda N, Kaisaki PJ et al: Mutations in the hepatocyte nuclear factor-1alpha gene in maturity-onset diabetes of the young (MODY3). Nature 1996; 384: 455-458.

52 Godart F, Bellanne-Chantelot C, Clauin S et al: Identification of seven novel nucleotide variants in the hepatocyte nuclear factor1alpha (TCF1) promoter region in MODY patients. Hum Mutat 2000; 15: 173-180.

53 Yang Q, Yamagata K, Fukui K et al: Hepatocyte nuclear factor1alpha modulates pancreatic beta-cell growth by regulating the expression of insulin-like growth factor-1 in INS-1 cells. Diabetes 2002; 51: 1785-1792.

54 Perneger TV: What's wrong with Bonferroni adjustments. BMJ 1998; 316: 1236-1238. 